\title{
Complete genome sequence analysis of canine bocavirus 1 identified for the first time in domestic cats
}

\author{
Jiangting Niu ${ }^{1} \cdot$ Shushuai Yi ${ }^{1} \cdot$ Hualei Wang ${ }^{2} \cdot$ Guoying Dong ${ }^{3} \cdot$ Yanli Zhao ${ }^{1} \cdot$ Yanbing Guo $^{1,4} \cdot$ Hao Dong ${ }^{5} \cdot$ Kai Wang $^{1}$. \\ Guixue $\mathrm{Hu}^{1}$
}

Received: 27 July 2018 / Accepted: 21 October 2018 / Published online: 28 November 2018

○) Springer-Verlag GmbH Austria, part of Springer Nature 2018

\begin{abstract}
In this study, we investigated the presence of canine bocaviruses (CBoVs) in fecal samples from 105 cats with diarrhea and 92 asymptomatic cats in northeast China. One fecal sample, 17CC0312, collected from an asymptomatic cat, was found to be positive for canine bocavirus $1(\mathrm{CBoV} 1)$. The nearly complete genome of this virus was cloned and sequenced. The viral genome was 5,069 nucleotides (nt) in length and combined four open reading frames (ORFs) in the order 5'-NS1-ORF4-NP1VP1/VP2-3'. The 17CC0312 virus shared more than 90.3\% nucleotide sequence identity with CBoV1 reference sequences and was placed within the $\mathrm{CBoV} 1$ group in a phylogenetic tree based on complete genome sequences. Further phylogenetic analysis based on the deduced amino acid sequence of the VP2 gene showed that this feline CBoV1 strain belongs to CBoV1 lineage 3. These data provide the first molecular evidence of the presence of $\mathrm{CBoV} 1$ in a domestic cat and suggest that cats might be carriers of CBoV1.
\end{abstract}

Bocaviruses, which are members of the genus Bocaparvovirus (formerly Bocavirus), subfamily Parvovirinae, family Parvoviridae, are small, non-enveloped, linear singlestranded DNA viruses [4]. In contrast to other parvoviruses, bocaviruses have an additional open reading frame (ORF)

Handling Editor: Sheela Ramamoorthy.

Jiangting Niu, Shushuai Yi and Hualei Wang contributed equally to this work.

\section{Hao Dong}

13504466861@163.com

$\triangle$ Guixue $\mathrm{Hu}$

huguixue901103@163.com

1 College of Animal Science and Technology, Jilin Agricultural University, Changchun, Jilin, China

2 Key Laboratory of Jilin Province for Zoonosis Prevention and Control, Military Veterinary Research Institute, Academy of Military Medical Sciences, Changchun, Jilin, China

3 College of Global Change and Earth System Science, Beijing Normal University, Beijing, China

4 Jilin Institute of Animal Husbandry and Veterinary Science, Changchun 130062, Jilin, China

5 College of Life Science, Jilin Agricultural University, Changchun, Jilin, China between the non-structural and structural coding regions, ORF1 and ORF2, encoding a phosphorylated non-structural protein, NP1 [4]. The original genus name Bocavirus was derived from the names of the first two members of the genus, bovine parvovirus (BPV) and canine minute virus (CnMV) [3, 16, 20]. However, because the list of viruses belonging to this genus is continually being updated with the discovery of novel viruses from different animal species, the International Committee on Taxonomy of Viruses (ICTV) has changed the taxonomic nomenclature for the family Parvoviridae. In the ICTV report updated in 2017 (https ://talk.ictvonline.org/taxonomy/), the genus Bocavirus was renamed Bocaparvovirus, and it now includes 21 officially recognized species: Carnivore bocaparvovirus 1 to 6 , Chiropteran bocaparvovirus 1 to 4 , Lagomorph bocaparvovirus 1, Pinniped bocaparvovirus 1 and 2, Primate bocaparvovirus 1 and 2 and Ungulate bocaparvovirus 1 to 6 . Bocaviruses can infect a variety of mammalian species, including humans, chimpanzees, gorillas, pigs, cows, bats, dogs, cats, rats, California sea lions, and pine martens $[4,15]$. Bocavirus infections typically cause diseases of the gastrointestinal and respiratory tract in their hosts. Disease symptoms tend to be more severe in young animals and humans, whereas subclinical infection is common in adults $[8,15]$. Worldwide epidemiological surveys have indicated that bocaviruses are widespread in their host species. 
Previous research has demonstrated that genetically diverse bocaviruses are present in cats, dogs and minks worldwide $[6,11,21,23,24]$. To date, six members of the genus Bocaparvovirus have been identified in carnivores. These viruses belong to the species Carnivore bocaparvovirus 1 to 6 , and include canine minute virus (CnMV), canine bocavirus 1 (CBoV1), feline bocavirus 1 (FBoV1), feline bocavirus 2 (FBoV2), feline bocavirus 3 (FBoV3) and mink bocavirus (MiBoV1), respectively (https://talk.ictvonline .org/taxonomy/). For better understanding, throughout this study we will use exemplar isolate names for each species. CnMV was the first bocavirus known to infect dogs, and it was first isolated from feces of healthy dogs in Germany in 1967 [1]. CnMV had been recognized to cause neonatal diseases and fertility disorders in dogs in the past 50 years [15]. In 2012, a novel canine bocavirus, CBoV1, was identified in respiratory samples from dogs, and the virus was associated with respiratory and enteric disease in dogs $[2,10]$. The third canine bocavirus, $\mathrm{CnBoV} 3$, which has not been officially assigned to a species), was discovered in canine liver in 2013 and only emerged in the United States [13]. The discovery of bocavirus infections in cats and minks occurred relatively late. In 2012, a novel bocavirus designated as FBoV-1 was first detected in Hong Kong [11]. Since then, two highly divergent feline bocaviruses, referred to as FBoV-2 and FBoV-3, were successively identified in the feces of cats [17, 26]. In 2016, a novel bocavirus, MiBoV1, was first identified in mink in China [23]. Although some clinical cases of bocavirus infections in carnivores had been reported, their association with disease remains controversial.

As companion animals, cats and dogs often share the same habitat, and interspecies transmission of viruses such as parvovirus, norovirus and influenza virus between them is not uncommon $[5,7,9]$. Transmission of bocaviruses between cats and dogs is likely due to frequent contact between animals of different species, there are no reports demonstrating that interspecies transmission of bocaviruses occurs between cats and dogs. In this study, we investigate the presence, circulation, and genetic characteristics of canine bocaviruses in domestic cats in northeast China and provide the first molecular evidence of the circulation of CBoV1 in domestic cats.

Fecal samples were collected from private veterinary clinics and animal shelters in northeast China between January 2016 and November 2017. Sample collection was done with the consent of the owners. In total, 197 fresh feces samples were collected from 105 cats with diarrhea and 92 asymptomatic cats. The samples were homogenized in phosphate-buffered saline solution (PBS) at a concentration of about $0.5 \mathrm{~g} / \mathrm{ml}$, and the supernatant was collected by centrifugation at $10,000 \times g$ for $10 \mathrm{~min}$. Genomic DNA was extracted using a Viral DNA Kit I (Omega, China) according the manufacturer's instructions. Canine bocavirus was detected by PCR using three different primer pairs targeting the NS1 genes of members of three $\mathrm{CBoV}$ species as described previously. The size of the amplified DNA fragment was $141 \mathrm{bp}$ for CnMV [11], $440 \mathrm{bp}$ for CBoV1 [6], and 386 bp for $\mathrm{CnBoV} 3$ [25]. The CBoV-positive samples were also examined for feline panleukopenia virus (FPV) [22], FBoV 1 to 3 [24], feline astrovirus (FeAstV) [17], feline kobuvirus (FeKoV) [14] and feline coronavirus (FCoV) [17] using previously described PCR/RT-PCR assays. Moreover, to determine the genome sequence of $\mathrm{CBoV} 1$, four pairs of previously described primers were used to amplify the complete nucleotide sequences [2]. DNA fragments of $938 \mathrm{bp}$, $1250 \mathrm{bp}, 1988 \mathrm{bp}$ and $1167 \mathrm{bp}$, respectively, were purified using an AxyPrep DNA Gel Extraction Kit (Axygen, China) and then cloned into the vectors PMD-18T (TaKaRa, China). At least three positive clones for each fragment were sent to Sangon Biotech (Shanghai, China) for Sanger sequencing.

The complete genome sequence was assembled using the SeqMan program, and ORFs were predicted using ORF Finder (https://www.ncbi.nlm.nih.gov/orffinder/) and confirmed using Gene Marks (http://opal.biology.gatech.edu/ GeneMark/genemarks.cgi). The complete genome sequence (5069 nt) and the deduced amino acid sequence (573 aa) of the VP2 gene generated from sample 17CC0312 in the present study and 28 reference sequences obtained from GenBank were aligned using the Clustal W method in DNAstar software, and a phylogenetic tree was constructed using the neighbor-joining method and a p-distance model with 1,000 bootstrap replicates in MEGA 7.0 as described previously by Choi et al. and Li et al. [3, 13].

Canine bocavirus DNA was detected in one fecal sample using specific primers targeting CBoV1. This positive sample (17CC0312) was collected from an asymptomatic cat (approximately 2 months old) from an animal shelter centre in Changchun in March 2017. Pairwise alignments based on a 440-nt region of the NS1 gene showed that $17 \mathrm{CC} 0312$ was closely related to $\mathrm{CBoV} 1$ reference sequences and shared $100 \%$ nucleotide identity with Korean isolate 14Q216 (KP281720). We also tested for other feline enteric viruses, including FPV, FBoV 1 to $3, \mathrm{FeAst}$, FeKoV and FCoV in sample 17CC0312, and the results were negative. Moreover, all of the fecal samples tested negative for CnMV and CnBoV3.

The nearly complete $\mathrm{CBoV}$ genome sequence has been deposited in the GenBank database under accession number MH626633. It is 5,069 nt in length and contains four ORFs. ORF1, ORF2 and ORF3 are predicted to encode a 667-aa non-structural protein (NS1), a 711-aa VP1/VP2 capsid protein, and a 195-aa NP1 protein, respectively. An additional ORF (ORF4) encoding an unknown 144-aa protein that was already reported in previous studies $[3,11]$ was found in the genome of 17CC0312, (Fig. 1). The genomic structure of $17 \mathrm{CC} 0312$ was coincident with that of $\mathrm{CBoV} 1$ 


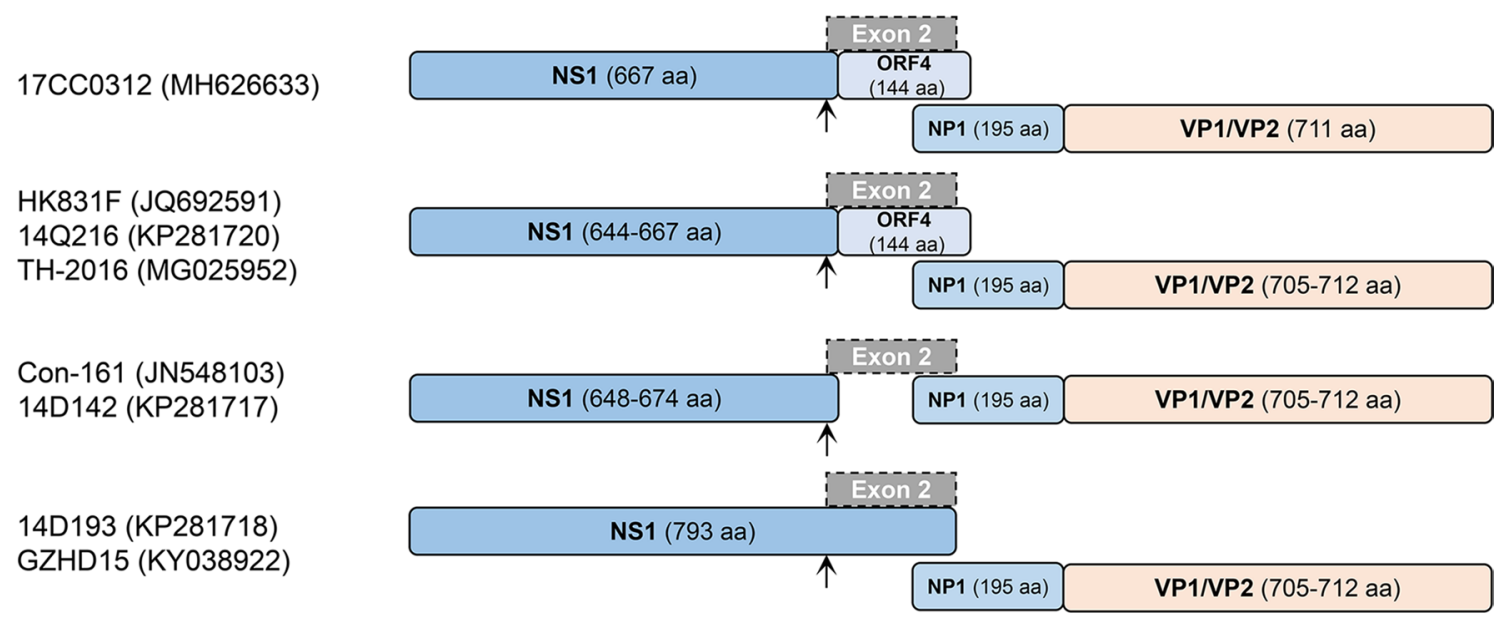

Fig. 1 Comparative genome organization of canine bocavirus 1 strain 17CC0312 identified in cats in this study and other reference strains of canine bocavirus 1. Grey boxes labelled "Exon 2" represent the second exon of the NS1 protein, and arrows indicate RNA-splicing sites

reference strains HK831F (JQ692591), 14Q216 (KP281720) and 14Q209 (KP281719). Recent studies have shown that CBoV1 strains have a second exon in their genome encoding the C-terminal region of NS1 and containing conserved RNA-splicing signals near the end of NS1, which may generate a longer NS1 protein [3]. ORF4, immediately downstream of ORF1, might also be generated by the second exon, but the splicing signals were changed to a cleavage signal. At present, the function of the ORF4-encoded protein is not known, but some investigations have found that it might enhance the pathogenicity of CBoV1 $[3,11]$. In this study, ORF4 was found in a CBoV1 strain identified in an asymptomatic cat, suggesting that the pathogenicity of CBoV1 might be related to the host. Further investigation is needed to confirm the relationship between the presence of ORF4 and the host range and pathogenicity of CBoV1.

The 17CC0312 isolate shared at least $90.3 \%$ nucleotide sequence identity with other $\mathrm{CBoV} 1$ reference sequences, including Con-161 (90.3\%, JN648103), TH-2016 (93.3\%, MG025952), 14Q209 (94.5\%, KP281719), 14Q216 (97.6\%, KP281720), and HK831F (97.9\%, JQ692591). However, when compared to CnMV, CnBoV3, and FBOV reference strains, the genome nucleotide sequence identity was less than $65 \%$. A neighbor-joining phylogenetic tree based on the full-length genome sequences showed that the 17CC0312 isolate belongs to the species Carnivore bocaparvovirus (Fig. 2a). Then, a similarity plot analysis based on the nearly complete genome sequence showed that all CBoV1 sequences were very similar to each other in the NS1 and NP1 regions, but less similar in the VP2 region (Fig. 3). A phylogenetic tree based on the deduced amino acid sequences of the complete VP2 gene of CBoVs showed that all CBoV1 strains grouped into three lineages and that $17 \mathrm{CC} 0312$ clustered within
CBoV1 lineage 3 (Fig. 2b). The complete VP2 protein of $17 \mathrm{CC} 0312$ was predicted to be at least $98.6 \%$ identical to those of the reference strains within CBoV1 lineage 3, including 14Q216 (KP281720), 13D250 (KP281716) and HK831F (JQ692591), at the amino acid level. Moreover, no unique amino acid changes were found in the genome of $17 \mathrm{CC} 0312$.

The parvovirus capsid protein VP2 plays a critical role in determining viral antigenicity, pathogenicity and host range. In previous studies, it had been demonstrated that changes in the host range of CPV are mainly dependent on a high rate of mutation and the positive selection of mutations in the VP2 protein [19]. Genetic diversity was also observed in the VP2 protein of CBoV1. Phylogenetic analysis suggested that $\mathrm{CBoV} 1$ strains belong to three different lineages, and CBoV1 strains within the same lineage possess stable and common mutations in the VP2 protein. These mutations might also be responsible for the change in host range of CBoV1. Moreover, it had been documented that CnMV can infect feline kidney cells in vitro $[12,18]$, suggesting that cats may be carriers of $\mathrm{CBoVs}$.

Cats and dogs are common companion animals that often live in close contact with each other. The frequent contact increases the probability of interspecies transmission of viruses. In the present study, $\mathrm{CBoV} 1$ strain $17 \mathrm{CC} 0312$ was identified in a domestic cat in an animal shelter that had adopted many cats and dogs. These animals had direct contact with each other daily, which might have resulted in the transmission of CBoVs from dogs to cats through the fecaloral route, as has been observed with canine parvovirus. CBoV1 was also found in fecal samples from two dogs at the same animal shelter with mild diarrhea. Pairwise alignments based on a portion of the VP2 gene showed that the two CBoV1 sequences identified in dogs were $99.9 \%$ identical 


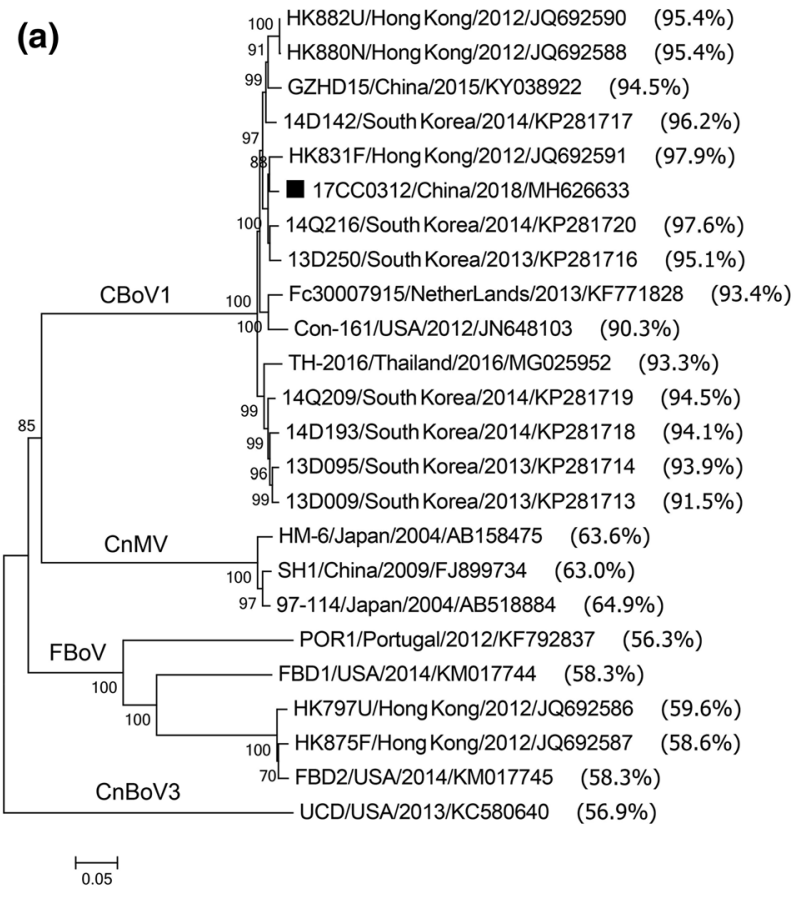

Fig. 2 Phylogenetic analysis based on the nucleotide sequence of fulllength genome (a) and the deduced amino acid sequences of the complete VP2 gene (b). A multiple alignments including the sequences of 17CC0312, canine bocavirus reference strains, and FBoV reference strains as an out-group were performed using the Clustal W program, and phylogenetic trees were constructed using the neighbour-joining method with 1,000 bootstrap replicates, and only bootstrap values $>70 \%$ are displayed above the tree branches. The strain names,

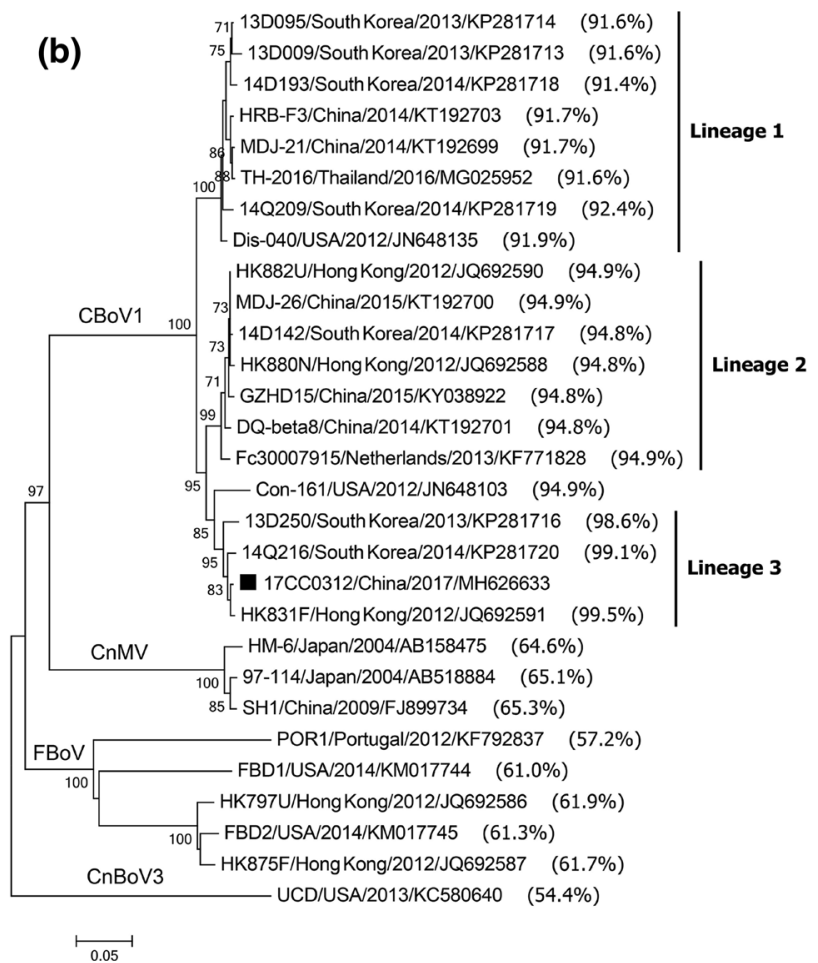

country, year of isolation, GenBank accession number, percent sequence identity (in parentheses) compared to 17CC0312, and the species of the canine bocavirus are shown in each phylogenetic tree. $\mathrm{CBoV}-2$ lineages proposed in the present study are only shown in the phylogenetic tree based on the complete VP2 protein sequence (b). A black square indicates $\mathrm{CBoV} 1$ strain $17 \mathrm{CC} 0312$ identified in cats in the present study

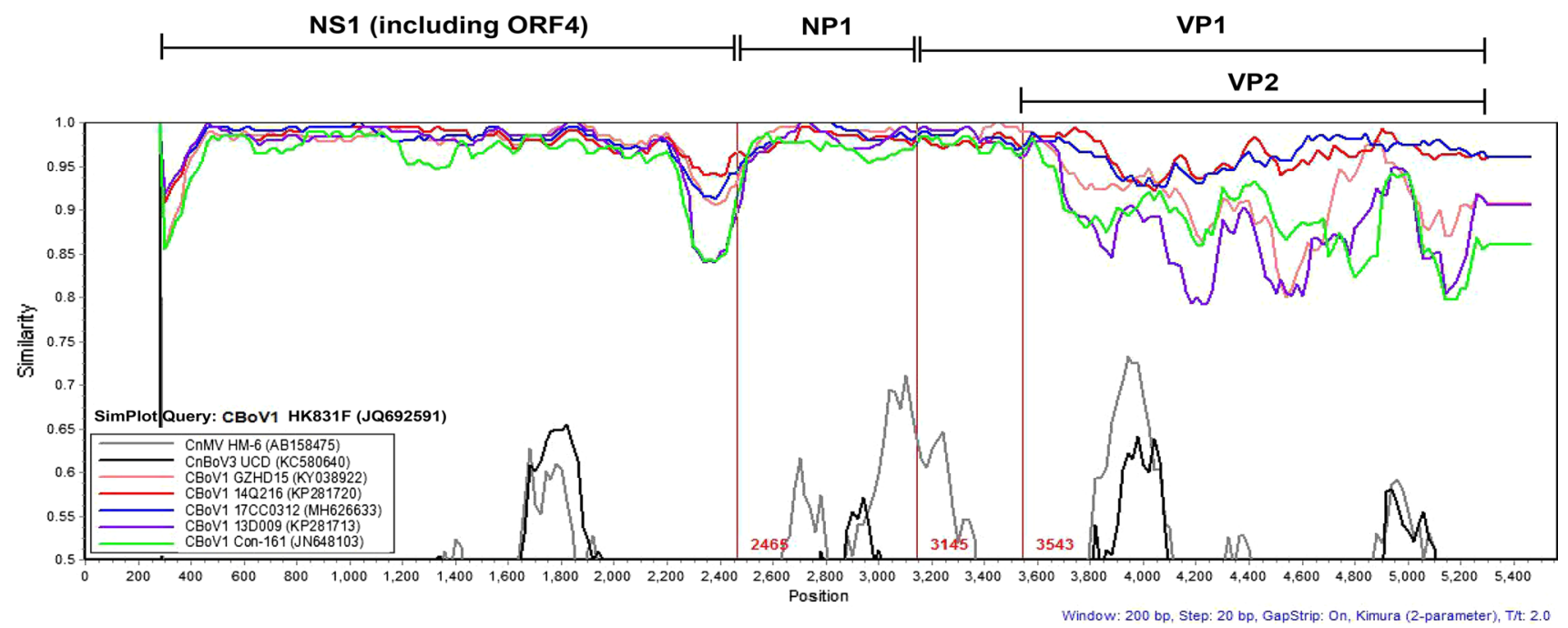

Fig. 3 Similarity plot analysis of the complete genome sequences of $17 \mathrm{CC} 0312$ (blue), four CBoV1 reference strains, including Con-161 (green), 13D009 (violet), 14Q216 (red) and GZHD15 (pink), CnMV strain HM-6 (gray) and $\mathrm{CnBoV} 3$ strain UCD (black) as out-group sequences, and $\mathrm{CBoV} 1$ strain $\mathrm{HK} 831 \mathrm{~F}$ as a query sequence, using the Kimura 2-parameter model with a sliding window of $200 \mathrm{nt}$ and a moving step size of $20 \mathrm{nt}$. The NS1, NP1, VP1 and VP2 regions are separated by red lines, and the nucleotide positions refer to the fulllength genome sequence of $\mathrm{CBoV} 1$ strain $\mathrm{HK} 831 \mathrm{~F}$ 
to $17 \mathrm{CC} 0312$ (data not shown), suggesting that the feline CBoV1 might have originated from these two dogs.

Collectively, our study provides the first molecular evidence of the presence of $\mathrm{CBoV} 1$ in cats and raises the possibility that cats may be carriers of CBoV1. Further investigations in more countries are needed to confirm that the infection with canine bocaviruses can occur in both cats and dogs.

Funding This study was supported by the Research Project of the National Key Research and Development Plan of China (Grant no. 2016YFD0501002). This study was also funded by the Special Public Welfare Industry Research of the Ministry of Agriculture of China (no. 201303042) and the National Key R\&D Program of China (no. 2017YFD0501703).

\section{Compliance with ethical standards}

Conflict of interest All authors declare no conflict of interest.

Ethical approval All animal experiments conformed to the guidelines and regulatory requirements established by the Animal Care and Use Committee of Jilin Agricultural University, Jilin Province, China. The owner's consent was obtained to collect samples from healthy and diarrheal cats.

\section{References}

1. Binn LN, Lazar EC, Eddy GA, Kajima M (1970) Recovery and characterization of a minute virus of canines. Infect Immun 1:503-508

2. Bodewes R, Lapp S, Hahn K, Habierski A, Forster C, Konig M, Wohlsein P, Osterhaus AD, Baumgartner W (2014) Novel canine bocavirus strain associated with severe enteritis in a dog litter. Vet Microbiol 174:1-8

3. Choi JW, Lee KH, Lee JI, Lee MH, Lee KK, Oem JK (2015) Genetic characteristics of canine bocaviruses in Korean dogs. Vet Microbiol 179:177-183

4. Cotmore SF, Agbandje-McKenna M, Chiorini JA, Mukha DV, Pintel DJ, Qiu J, Soderlund-Venermo M, Tattersall P, Tijssen P, Gatherer D, Davison AJ (2014) The family Parvoviridae. Arch Virol 159:1239-1247

5. Di Martino B, Di Profio F, Melegari I, Sarchese V, Cafiero MA, Robetto S, Aste G, Lanave G, Marsilio F, Martella V (2016) A novel feline norovirus in diarrheic cats. Infect Genet Evol 38:132-137

6. Guo D, Wang Z, Yao S, Li C, Geng Y, Wang E, Zhao X, Su M, Wei S, Wang X, Feng L, Chang YF, Sun D (2016) Epidemiological investigation reveals genetic diversity and high co-infection rate of canine bocavirus strains circulating in Heilongjiang province, Northeast China. Res Vet Sci 106:7-13

7. Ikeda Y, Nakamura K, Miyazawa T, Takahashi E, Mochizuki M (2002) Feline host range of canine parvovirus: recent emergence of new antigenic types in cats. Emerg Infect Dis 8:341-346

8. Jartti T, Hedman K, Jartti L, Ruuskanen O, Allander T, SoderlundVenermo M (2012) Human bocavirus-the first 5 years. Rev Med Virol 22:46-64
9. Jeoung HY, Lim SI, Shin BH, Lim JA, Song JY, Song DS, Kang BK, Moon HJ, An DJ (2013) A novel canine influenza H3N2 virus isolated from cats in an animal shelter. Vet Microbiol 165:281-286

10. Kapoor A, Mehta N, Dubovi EJ, Simmonds P, Govindasamy L, Medina JL, Street C, Shields S, Lipkin WI (2012) Characterization of novel canine bocaviruses and their association with respiratory disease. J Gen Virol 93:341-346

11. Lau SK, Woo PC, Yeung HC, Teng JL, Wu Y, Bai R, Fan RY, Chan KH, Yuen KY (2012) Identification and characterization of bocaviruses in cats and dogs reveals a novel feline bocavirus and a novel genetic group of canine bocavirus. J Gen Virol 93:1573-1582

12. Li F, Zhang Q, Yao Q, Chen L, Li J, Qiu J, Sun Y (2014) The DNA replication, virogenesis and infection of canine minute virus in non-permissive and permissive cells. Virus Res 179:147-152

13. Li L, Pesavento PA, Leutenegger CM, Estrada M, Coffey LL, Naccache SN, Samayoa E, Chiu C, Qiu J, Wang C, Deng X, Delwart E (2013) A novel bocavirus in canine liver. Virol J 10:54

14. Lu G, Zhang X, Luo J, Sun Y, Xu H, Huang J, Ou J, Li S (2018) First report and genetic characterization of feline kobuvirus in diarrhoeic cats in China. Transbound Emerg Dis 65:1357-1363

15. Manteufel J, Truyen U (2008) Animal bocaviruses: a brief review. Intervirology 51:328-334

16. Mochizuki M, Hashimoto M, Hajima T, Takiguchi M, Hashimoto A, Une Y, Roerink F, Ohshima T, Parrish CR, Carmichael LE (2002) Virologic and serologic identification of minute virus of canines (canine parvovirus type 1) from dogs in Japan. J Clin Microbiol 40:3993-3998

17. Ng TF, Mesquita JR, Nascimento MS, Kondov NO, Wong W, Reuter G, Knowles NJ, Vega E, Esona MD, Deng X, Vinje J, Delwart E (2014) Feline fecal virome reveals novel and prevalent enteric viruses. Vet Microbiol 171:102-111

18. Pratelli A, Moschidou P (2012) Host range of Canine minute virus in cell culture. J Vet Diagn Investig 24:981-985

19. Shackelton LA, Parrish CR, Truyen U, Holmes EC (2005) High rate of viral evolution associated with the emergence of carnivore parvovirus. Proc Natl Acad Sci USA 102:379-384

20. Storz J, Leary JJ, Carlson JH, Bates RC (1978) Parvoviruses associated with diarrhea in calves. J Am Vet Med Assoc 173:624-627

21. Takano T, Takadate Y, Doki T, Hohdatsu T (2016) Genetic characterization of feline bocavirus detected in cats in Japan. Arch Virol 161:2825-2828

22. Yang S, Wang S, Feng H, Zeng L, Xia Z, Zhang R, Zou X, Wang C, Liu Q, Xia X (2010) Isolation and characterization of feline panleukopenia virus from a diarrheic monkey. Vet Microbiol 143:155-159

23. Yang S, Wang Y, Li W, Fan Z, Jiang L, Lin Y, Fu X, Shen Q, Sun Z, Wang X, Deng X, Zhang W, Delwart E (2016) A novel bocavirus from domestic mink, China. Virus Genes 52:887-890

24. Yi S, Niu J, Wang H, Dong G, Zhao Y, Dong H, Guo Y, Wang K, Hu G (2018) Detection and genetic characterization of feline bocavirus in Northeast China. Virol J 15:125

25. Zhai SL, Lin T, Chen YW, Liu R, Lv DH, Wen XH, Zhu XL, Wang D, Li F, Wei WK (2017) First complete genome sequence of canine bocavirus 2 in mainland China. New Microbes New Infect 18:47-49

26. Zhang W, Li L, Deng X, Kapusinszky B, Pesavento PA, Delwart E (2014) Faecal virome of cats in an animal shelter. J Gen Virol 95:2553-2564 